\title{
Polarization Measurements in High-Energy Deuteron Photodisintegration
}

K. Wijesooriya, ${ }^{10, *}$ A. Afanasev, ${ }^{20,26}$ M. Amarian, ${ }^{11}$ K. Aniol, ${ }^{3}$ S. Becher, ${ }^{9}$ K. Benslama, ${ }^{23}$ L. Bimbot, ${ }^{22}$ P. Bosted, ${ }^{16}$ E. Brash, ${ }^{23}$ J. Calarco, ${ }^{18}$ Z. Chai, ${ }^{17}$ C. C. Chang, ${ }^{15}$ T. Chang, ${ }^{10}$ J. P. Chen, ${ }^{26}$ S. Choi, ${ }^{25}$ E. Chudakov, ${ }^{26}$ S. Churchwell, ${ }^{6}$ D. Crovelli, ${ }^{24}$ S. Dieterich,${ }^{24}$ S. Dumalski ${ }^{23}$ D. Dutta, ${ }^{17}$ M. Epstein, ${ }^{3}$ K. Fissum, ${ }^{14}$ B. Fox,${ }^{4}$ S. Frullani, ${ }^{11}$ H. Gao, ${ }^{17}$

J. Gao, ${ }^{2}$ F. Garibaldi, ${ }^{11}$ O. Gayou, ${ }^{1,28}$ R. Gilman, ${ }^{24,26, \dagger}$ S. Glamazdin, ${ }^{13}$ C. Glashausser ${ }^{24}$ J. Gomez, ${ }^{26}$ V. Gorbenko, ${ }^{13}$ O. Hansen, ${ }^{26}$ R. J. Holt,,${ }^{10} *$ J. Hovdebo,${ }^{23}$ G. M. Huber, ${ }^{23}$ C. W. de Jager, ${ }^{26}$ X. Jiang, ${ }^{24}$ C. Jones, ${ }^{2}$ M. K. Jones, ${ }^{21}$ J. Kelly, ${ }^{15}$ E. Kinney, ${ }^{4}$ E. Kooijman, ${ }^{12}$ G. Kumbartzki, ${ }^{24}$ M. Kuss, ${ }^{26}$ J. LeRose, ${ }^{26}$ M. Liang, ${ }^{26}$ R. Lindgren, ${ }^{27}$ N. Liyanage, ${ }^{26}$ S. Malov, ${ }^{24}$ D. J. Margaziotis, ${ }^{3}$ P. Markowitz, ${ }^{7}$ K. McCormick, ${ }^{5}$ D. Meekins, ${ }^{8}$ Z.-E. Meziani, ${ }^{25}$ R. Michaels, ${ }^{26}$ J. Mitchell, ${ }^{26}$ L. Morand, ${ }^{24}$ C. F. Perdrisat,${ }^{28}$ R. Pomatsalyuk, ${ }^{13}$ V. Punjabi, ${ }^{19}$ R. D. Ransome, ${ }^{24}$ R. Roche,${ }^{8}$ M. Rvachev, ${ }^{17}$ A. Saha, ${ }^{26}$ A. Sarty, ${ }^{8,}$ E. C. Schulte, ${ }^{10}$ D. Simon, ${ }^{9}$ S. Strauch, ${ }^{24}$ R. Suleiman, ${ }^{12}$ L. Todor,${ }^{21}$ P. E. Ulmer, ${ }^{21}$ G. M. Urciuoli, ${ }^{11}$ B. Wojtsekhowski, ${ }^{26}$ F. Xiong, ${ }^{17}$ and W. Xu ${ }^{17}$

\author{
(Jefferson Lab Hall A Collaboration) \\ ${ }^{1}$ Université Blaise Pascal/IN2P3, F-63177 Aubière, France \\ ${ }^{2}$ California Institute of Technology, Pasadena, California 91125 \\ ${ }^{3}$ California State University, Los Angeles, California 90032 \\ ${ }^{4}$ University of Colorado, CB 446, Boulder, Colorado 80309 \\ ${ }^{5}$ DAPNIA, Saclay, France \\ ${ }^{6}$ Duke University, Durham, North Carolina 27706 \\ ${ }^{7}$ Florida International University, Miami, Florida 33199 \\ ${ }^{8}$ Florida State University, Tallahassee, Florida 32306 \\ ${ }^{9}$ University of Georgia, Athens, Georgia 30602 \\ ${ }^{10}$ University of Illinois, Urbana-Champaign, Illinois 61801 \\ ${ }^{11}$ INFN, Sezione Sanitá and Istituto Superiore di Sanitá, Laboratorio di Fisica, I-00161 Rome, Italy \\ ${ }^{12}$ Kent State University, Kent, Ohio 44242 \\ ${ }^{13}$ Kharkov Institute of Physics and Technology, Kharkov 310108, Ukraine \\ ${ }^{14}$ University of Lund, P.O. Box 118, S-221 00 Lund, Sweden \\ ${ }^{15}$ University of Maryland, College Park, Maryland 20742 \\ ${ }^{16}$ University of Massachusetts, Amherst, Massachusetts 01003 \\ ${ }^{17}$ Massachusetts Institute of Technology, Cambridge, Massachusetts 02139 \\ ${ }^{18}$ University of New Hampshire, Durham, New Hampshire 03824 \\ ${ }^{19}$ Norfolk State University, Norfolk, Virginia 23504 \\ ${ }^{20}$ North Carolina Central University, Durham, North Carolina 27707 \\ ${ }^{21}$ Old Dominion University, Norfolk, Virginia 23529 \\ ${ }^{22}$ Institut de Physique Nucléaire, F-91406 Orsay, France \\ ${ }^{23}$ University of Regina, Regina, Saskatchewan, Canada S4S OA2 \\ ${ }^{24}$ Rutgers, The State University of New Jersey, Piscataway, New Jersey 08854 \\ ${ }^{25}$ Temple University, Philadelphia, Pennsylvania 19122 \\ ${ }^{26}$ Thomas Jefferson National Accelerator Facility, Newport News, Virginia 23606 \\ ${ }^{27}$ University of Virginia, Charlottesville, Virginia 22901 \\ ${ }^{28}$ College of William and Mary, Williamsburg, Virginia 23187
}

(Received 15 September 2000; revised manuscript received 19 December 2000)

\begin{abstract}
We present measurements of the recoil proton polarization for the $d(\vec{\gamma}, \vec{p}) n$ reaction at $\theta_{\text {c.m. }}=90^{\circ}$ for photon energies up to $2.4 \mathrm{GeV}$. These are the first data in this reaction for polarization transfer with circularly polarized photons. The induced polarization $p_{y}$ vanishes above $1 \mathrm{GeV}$, contrary to meson-baryon model expectations, in which resonances lead to large polarizations. However, the polarization transfer $C_{x}$ does not vanish above $1 \mathrm{GeV}$, inconsistent with hadron helicity conservation. Thus, we show that the scaling behavior observed in the $d(\gamma, p) n$ cross sections is not a result of perturbative QCD. These data should provide important tests of new nonperturbative calculations in the intermediate energy regime.
\end{abstract}

DOI: 10.1103/PhysRevLett.86.2975

One of the central goals of nuclear physics is to determine the role of quarks and gluons in nuclei and in nuclear reactions. Traditionally, nuclei and nuclear reactions are
PACS numbers: 25.20.-x, 24.70.+s, 24.85.+p, 25.10.+s

described in terms of effective nucleon-nucleon interactions which are mediated by the exchange of mesons. Meson-baryon model (MBM) calculations have been 
extended into the $\mathrm{GeV}$ region by explicitly including excited nucleon states. At very high energies, perturbative quantum chromodynamics (pQCD) is expected to apply. Nonperturbative QCD calculations are required in the intermediate regime where neither the MBM nor pQCD apply.

Polarization studies in electron-deuteron elastic scattering have proved to be a powerful method to distinguish among various descriptions of the underlying reaction mechanism. Measurements of $t_{20}$ have shown conclusively for a momentum transfer less than $Q^{2}=1.7 \mathrm{GeV}^{2}$ that only MBMs describe electron-deuteron scattering [1]. However, above $2 \mathrm{GeV}^{2}$, where no polarization data exist, the deuteron form factor is consistent with both MBMs and a nonperturbative QCD model [2].

Similarly, the deuteron photodisintegration cross sections [3] at $\theta_{\text {c.m. }}=90^{\circ}$ and photon energies above $1 \mathrm{GeV}$, which are equivalent to $Q^{2}=4 \mathrm{GeV}^{2}$ in $e-d$ elastic scattering [4], exhibit an $s^{-11}$ scaling behavior that is consistent with a MBM [5], pQCD [6], and a nonperturbative QCD model [7]. For this reason, we now provide new polarization data up to $2.4 \mathrm{GeV}$ (equivalent to $9.6 \mathrm{GeV}^{2}$ in $e-d$ scattering) to provide a stringent test of these competing pictures of deuteron photodisintegration. We show that $\mathrm{pQCD}$ is not a valid description of deuteron photodisintegration below $2.4 \mathrm{GeV}$. However, we also show that a state-of-the-art meson exchange calculation, which describes the cross section data, fails to even qualitatively describe the induced polarization above a photon energy of about $1 \mathrm{GeV}$.

There are two commonly accepted signatures of pQCD in exclusive reactions. First, the energy dependence of the cross section should be consistent with the constituent counting rules (CCR) [6]. Many reactions are consistent with the CCR [8], including deuteron photodisintegration at $\theta_{\text {c.m. }}=90^{\circ}[3,9-11]$. Second, the polarization observables should be consistent with hadron helicity conservation (HHC) [12]. HHC arises from the fact that vector interactions (photon or gluon coupling with the quarks) conserve chirality, leading to conservation of the sum of the components of the hadronic spins along their respective momentum directions, and to predictions of spin observables. No exclusive reactions have been shown to satisfy HHC, but the number of studies is limited. The most detailed investigation was for proton-proton elastic scattering [13], which is approximately consistent with CCR, but not with HHC. This has been attributed to long distance phenomena in which there are three independent scatterings of quarks in the beam proton from quarks in the target proton $[14,15]$. This long distance (Landshoff) mechanism is strongly suppressed in photoreactions because the incoming photon can interact only with a single quark in the target [16]. The present work is the first direct test of HHC in a photoreaction in a kinematic region where the cross section exhibits scaling behavior.

The measurements were performed at the Thomas Jefferson National Accelerator Facility (JLab) Hall A. A polarized electron beam source used a strained GaAs crystal to produce $\mathrm{a} \approx 30 \mu \mathrm{A}$ polarized electron beam. The beam helicity state was flipped pseudorandomly at $30 \mathrm{~Hz}$. Charge asymmetries between the two helicity states were negligible. The beam polarization $P_{e}$ was measured every few days with the Hall A Møller polarimeter, and averaged about $70 \%$, with typical uncertainties of $\pm 0.3 \%$ (stat) and $\pm 3.0 \%$ (syst).

Circularly polarized bremsstrahlung photons were generated when the electron beam impinged on a copper radiator with a thickness of $6 \%$ of a radiation length ( $4 \%$ for the lowest energy, $0.5 \mathrm{GeV}$ ), positioned $\approx 73 \mathrm{~cm}$ upstream of a $15 \mathrm{~cm}$ liquid deuterium target. The ratio of the photon polarization $P_{\gamma}$ to the beam polarization can be directly calculated [17]; for the near-end-point photons of our experimental conditions, $P_{\gamma} / P_{e}$ is $98.4 \%-99.8 \%$.

Protons from the target were detected in the Hall A high resolution hadron spectrometer. The trajectories were measured with vertical drift chambers (VDCs) located in the focal plane. The scattering angles, momentum, and interaction position at the target were calculated from the VDC tracks. Two planes of plastic scintillators provided triggering and time-of-flight information for particle identification. An aerogel Čerenkov counter was used to identify and reject pions, which constitute a $1 \%$ background in these measurements.

The final element in the detector stack was the proton polarimeter, consisting of front and rear straw chambers that determined the scattering angles in a carbon analyzer. Transverse components of the proton polarization in the focal plane led to azimuthal asymmetries in the scattering distribution. All triggering scintillators were located before the analyzer, to prevent possible false asymmetries, at the expense of reading in events in which the protons were absorbed in the analyzer. The efficiency was large because of the large rear chambers, which detected all protons scattered at angles less than $15^{\circ}$, and had high geometric acceptance for scatterings up to $30^{\circ}$ in the analyzer. The induced (transferred) polarization was determined by the sum (difference) of the azimuthal asymmetry distributions for the two beam helicity states.

The determination and subtraction of background processes, from electrodisintegration and from interactions with the target cell walls, were very similar to the procedures used for previous cross section measurements [3,9-11]. Additional backgrounds included cosmic rays and neutral particles. Cosmic rays were easily rejected with scintillator time-of-flight cuts. Neutral particles interacted with the scintillators to generate triggers, but left no tracks in the VDC or polarimeter chambers. The incident photon energy was reconstructed from the scattered proton energy and angle, using two-body photodisintegration kinematics. Only events between the bremsstrahlung end point and the pion production threshold were used in the analysis.

Determination of the proton polarization required a careful treatment of polarimeter systematics. The alignment 
of the chambers was determined with straight-through trajectories, with the carbon analyzer removed. Additional systematic studies were performed with $\vec{e} p$ elastic scattering measurements at each of the eight spectrometer momentum settings used for the data of this experiment, since the analyzer thickness was varied to optimize the figure of merit for each setting. Polarization transfer in $\vec{e} p \rightarrow e \vec{p}$ allows determination of both the ratio of the proton's electromagnetic form factors [18] and the carbon analyzing power, after accounting for spin transport through the spectrometer and the beam polarization. The spin transport analysis is similar to that of [19]. Our measurements [20] agree well with previous data for the form factor ratio [19] and for the carbon analyzing power $[19,21,22]$. The induced polarization in ep elastic scattering vanishes - neglecting small effects from two-photon exchange-allowing a direct measurement of the false asymmetries in the polarimeter. Cone tests largely ensure that false asymmetries are small, with magnitude typically $<0.01$ and a smooth variation across the acceptance. The photodisintegration data for the induced polarization are corrected for the measured false asymmetries. For the polarization transfer data, the false asymmetries largely cancel with the helicity difference.

Figure 1 compares our new data for the normal component of the induced polarization $p_{y}$ [23] to previous measurements [24-28] and theoretical predictions. While our low-energy data are in good agreement with other previous measurements, the disagreement of our data with the

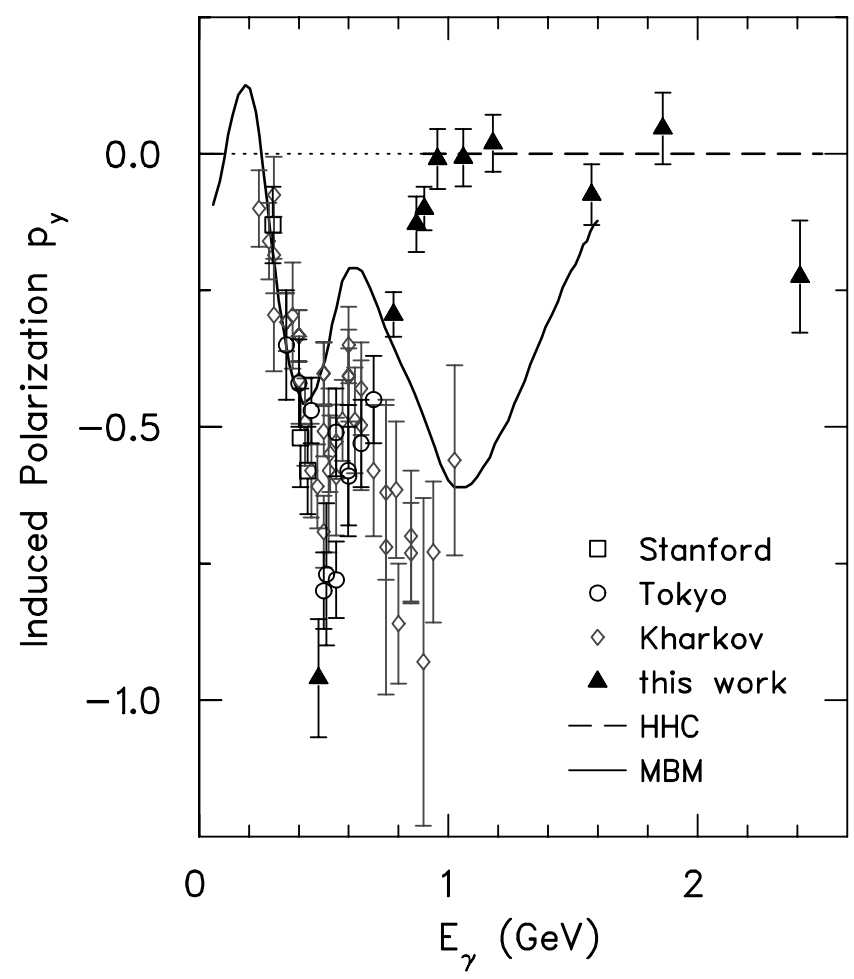

FIG. 1. Induced polarization $p_{y}$ in deuteron photodisintegration at $\theta_{\text {c.m. }}=90^{\circ}$. Only statistical uncertainties are shown. The curves are described in the text. highest-energy Kharkov measurements [28] is evident. Note that the Kharkov measurements were taken under difficult experimental conditions, with large background rates, and without a polarized beam, which would have allowed a polarimeter calibration.

Above about $1 \mathrm{GeV}$, the induced polarization is small, consistent with zero. While the $2.4 \mathrm{GeV}$ point might indicate a deviation from zero, the six highest-energy points are consistent with zero, with $\chi^{2} /$ d.o.f. $=1.1$, not including systematic uncertainties. The average of the six points is $-0.02 \pm 0.02$. Analysis of sensitivity to different false asymmetry models, alignment/tracking procedures, cuts, spin transport, and uncertainties in the carbon analyzing power leads to an estimated systematic uncertainty of about \pm 0.04 for these induced polarizations. The largest contribution is \pm 0.03 from the false asymmetry systematics.

The consistency of our measurements above $1 \mathrm{GeV}$ with the $\mathrm{pQCD}$ helicity conservation prediction that $p_{y}=0$ (dashed line, HHC), and the disagreement with the Bonn MBM prediction (solid line, MBM) [29] is striking. It is often suggested that at high momentum transfers/short distances, the MBM picture will break down and quark models will be needed. In our data, $p_{y}$ vanishes when $-t \approx 1 \mathrm{GeV}^{2}$. Do the qualitatively different characters of the data and calculation near and above $1 \mathrm{GeV}$ indicate a possible breakdown? The large values of $p_{y}$ predicted by the Bonn group are similar to the predictions of other existing calculations - see below - which involve a number of approximations. Further theoretical studies are needed to come to a firm conclusion. The data only conclusively indicate that the existing calculations are not adequate.

The Bonn calculation includes $\pi, \rho, \eta$, and $\omega$ exchanges, plus all well-established nucleon and $\Delta$ resonances with mass less than $2 \mathrm{GeV}$ and $J \leq 5 / 2$. Predictions in the $\Delta$ resonance region are similar to those of [30-32], which included only the $\Delta$ resonance, as well as to those of [33], which also included the Roper, $S_{11}$, and $D_{13}$ resonances. The calculations suggest that final state interaction effects are small and that the Born amplitude is nearly real. At their peaks, the resonance amplitudes are imaginary in phase, and the stronger resonance amplitudes, of the $\Delta, D_{1,3}$, and $D_{3,3}$, are similar in magnitude to the Born amplitude. The predicted induced polarization then becomes large near the resonance peaks, much larger than is observed in the data near $1 \mathrm{GeV}$.

The induced polarization $p_{y}$ is given - rewriting the expression from [27] - by

$$
f(\theta) p_{y}=2 \operatorname{Im} \sum_{i=1}^{3}\left[F_{i,+}^{*} F_{i+3,-}+F_{i,-} F_{i+3,+}^{*}\right],
$$

with $f(\theta)=\sum_{i=1}^{6} \sum_{ \pm}\left|F_{i, \pm}\right|^{2}$. The four amplitudes that conserve hadronic helicity, $F_{1,+}, F_{3,-}, F_{5,+}$, and $F_{5,-}$, are multiplied by amplitudes that do not conserve hadronic helicity. While HHC would naturally explain the vanishing 
of $p_{y}$, other explanations are possible, such as the two amplitudes in each product term having the same phase. Further tests of HHC can be provided by polarization transfer coefficients.

The transverse in-plane polarization transfer $C_{x}$ results from the real part of the same combination of amplitudes as $p_{y}$. While $C_{x}$ will vanish if there is HHC, it does not necessarily vanish if the amplitudes have the same phase. The longitudinal in-plane polarization transfer $C_{z}$ is given by

$$
f(\theta) C_{z}=\sum_{i=1}^{6} \sum_{ \pm} \pm\left|F_{i, \pm}\right|^{2}
$$

With HHC and the assumptions of [34] about relations between the helicity conserving amplitudes at $\theta_{\text {c.m. }}=90^{\circ}$, $C_{z}$ also vanishes.

Our data for $C_{x}$ and $C_{z}$ are shown in Fig. 2; no previous data, and no calculations, exist for these observables. Systematic uncertainties for these data are in the range 0.010.03 , except the $2.4 \mathrm{GeV} C_{x}$ and $1.9 \mathrm{GeV} C_{z}$ data points have systematic uncertainties of 0.06 . No points are shown at $1.1 \mathrm{GeV}$, because an unpolarized beam was used at this energy, and at $2.4 \mathrm{GeV}$ for $C_{z}$, due to the unfavorable spin transport which makes the uncertainty $\approx 1$. The longitudinal component $C_{z}$ appears to decrease with energy above $1 \mathrm{GeV}$, and could become consistent with zero near $2 \mathrm{GeV}$,

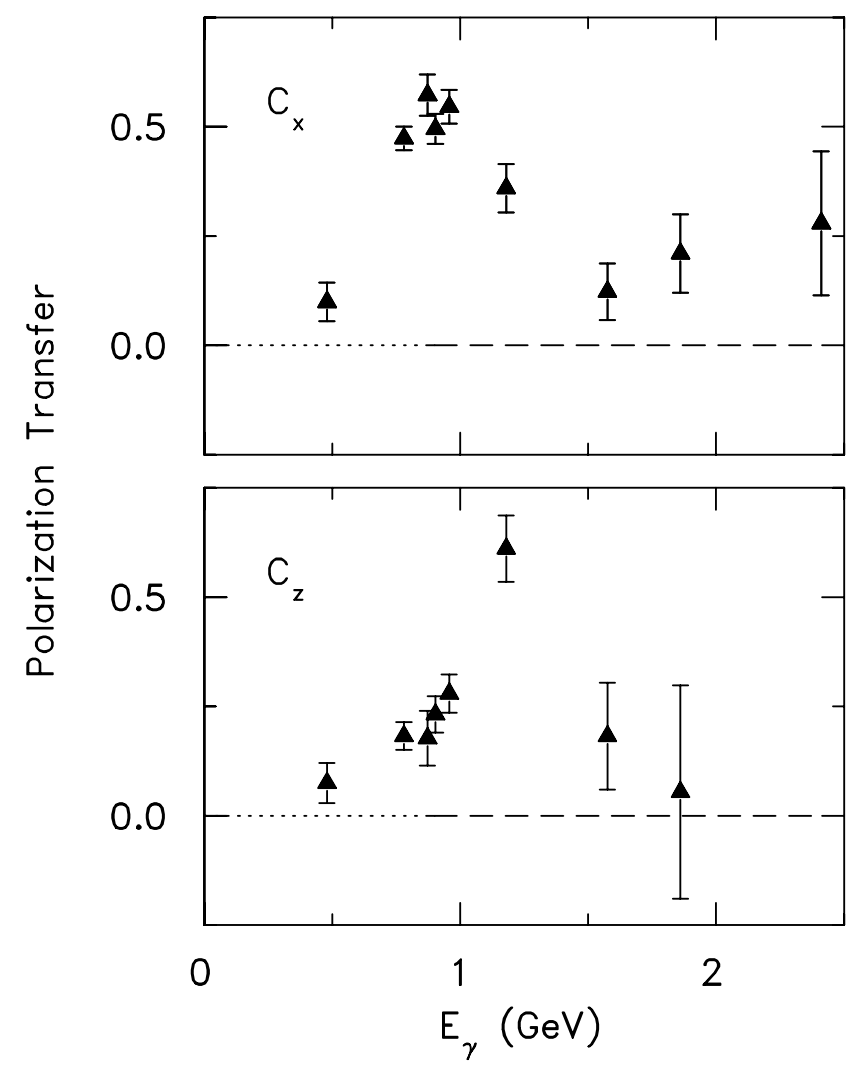

FIG. 2. Polarization transfers $C_{x}$ and $C_{z}$ in deuteron photodisintegration at $\theta_{\text {c.m. }}=90^{\circ}$. Only statistical uncertainties are shown. but uncertainties are large. The transverse component $C_{x}$ is nonzero in the energy range of this experiment, showing clearly that HHC is not valid, and thus pQCD cannot account for the observed data [35].

We have shown that existing MBM and $\mathrm{pQCD}$ predictions fail for the polarization data. We now consider several nonperturbative quark models, which have been applied to deuteron photodisintegration cross sections. The QCD rescattering model [7] provides absolute cross section calculations that agree with the data. The nonperturbative contributions are effectively included in the hard scattering $p n$ amplitude, which is taken from the $p n$ data. Preliminary induced polarization calculations [36] from this model indicate that $p_{y}$ is small, for energies above about $2.5 \mathrm{GeV}$, as it is in the present data for energies from 1 to $2.4 \mathrm{GeV}$. There are also cross section calculations in the quark-gluon string model [37], and polarization calculations are underway [38]. The quark exchange model [39] suggests that the amplitudes are nearly real; this can explain $p_{y}$, but calculations are needed for $C_{x}$ and $C_{z}$. The reduced nuclear amplitudes model [40] does not reproduce the cross sections well, and assumes helicity conservation, which would incorrectly predict that $C_{x}$ vanishes.

To summarize, we provide new benchmark data for recoil polarization in deuteron photodisintegration. Existing meson-baryon models fail to describe the data for the induced polarizations, which are surprisingly small for energies above about $1 \mathrm{GeV}$. The polarization transfer data are inconsistent with hadron helicity conservation, which is generally expected from $\mathrm{pQCD}$. These data should provide important tests of new nonperturbative calculations in the intermediate energy regime.

We thank G. Farrar, N. Isgur, G. A. Miller, A. Radyushkin, J. Ralston, and M. Sargsian for many interesting discussions. We thank M. Sargsian for allowing us to mention his results prior to publication. We thank the JLab physics and accelerator divisions for their support, especially Ed Folts and the Hall A technical staff, and Charlie Sinclair and the polarized source group. This work was supported by the U.S. Department of Energy, the U.S. National Science Foundation, the Natural Sciences and Engineering Research Council of Canada, the French Commissariat à l'Energie Atomique and Centre National de la Recherche Scientifique, the Italian National Institute for Nuclear Physics, and the Swedish Natural Science Research Council. The Southeastern Universities Research Association (SURA) operates the Thomas Jefferson National Accelerator Facility under DOE Contract No. DE-AC05-84ER40150. The polarimeter was funded by the U.S. National Science Foundation, Grants No. PHY 9213864 and No. PHY 9213869.

\footnotetext{
*Present address: Argonne National Laboratory, Argonne, IL 60439.

${ }^{\dagger}$ Corresponding author.

Email address: gilman@jlab.org
} 
†Present address: St. Mary’s University, Halifax, Nova Scotia, Canada B3H 3C3.

[1] D. Abbott et al., Phys. Rev. Lett. 84, 5053 (2000).

[2] L. C. Alexa et al., Phys. Rev. Lett. 82, 1374 (1999).

[3] C. Bochna et al., Phys. Rev. Lett. 81, 4576 (1998).

[4] R. Holt, Phys. Rev. C 41, 2400 (1990).

[5] A. E. L. Dieperink and S. I. Nagorny, Phys. Lett. B 456, 9 (1999).

[6] S. J. Brodsky and G. R. Farrar, Phys. Rev. Lett. 31, 1153 (1973); V. Matveev et al., Nuovo Cimento Lett. 7, 719 (1973).

[7] L. L. Frankfurt et al., Phys. Rev. Lett. 84, 3045 (2000); L. L. Frankfurt et al., Nucl. Phys. A663, 349 (2000).

[8] R. L. Anderson et al., Phys. Rev. D 14, 679 (1976).

[9] J. E. Belz et al., Phys. Rev. Lett. 74, 646 (1995).

[10] S. J. Freedman et al., Phys. Rev. C 48, 1864 (1993).

[11] J. Napolitano et al., Phys. Rev. Lett. 61, 2530 (1988).

[12] See S. J. Brodsky and G. P. Lepage, Phys. Rev. D 24, 2848 (1981), and references therein.

[13] D. G. Crabb et al., Phys. Rev. Lett. 65, 3241 (1990).

[14] T. Gousset, B. Pire, and J. P. Ralston, Phys. Rev. D 53, 1202 (1996).

[15] C. Carlson and M. Chachkhunashvili, Phys. Rev. D 45, 2555 (1992).

[16] A. Afanasev, C. Carlson, and C. Wahlquist, Phys. Rev. D 61, 034014 (2000).

[17] H. Olsen and L. C. Maximon, Phys. Rev. 110, 589 (1958).

[18] A. I. Akhiezer and M. P. Rekalo, Sov. J. Part. Nucl. 3, 277 (1974); R. Arnold, C. Carlson, and F. Gross, Phys. Rev. C 23, 363 (1981).

[19] M. K. Jones et al., Phys. Rev. Lett. 84, 1398 (2000); V. Punjabi et al. (to be published).

[20] K. Wijesooriya et al. (to be published).

[21] M. W. McNaughton et al., Nucl. Instrum. Methods Phys. Res., Sect. A 241, 435 (1985).

[22] N.E. Cheung et al., Nucl. Instrum. Methods Phys. Res., Sect. A 363, 561 (1995); B. Bonin et al., Nucl. Instrum. Methods Phys. Res., Sect. A 288, 379 (1990).

[23] With photon momentum $\vec{k}$ and proton momentum $\vec{q}$, the coordinate system is $\hat{z}=\hat{q}, \hat{y}=\hat{k} \times \hat{q}$, and $\hat{x}=\hat{y} \times \hat{z}$.
[24] F. F. Liu et al., Phys. Rev. 165, 1478 (1968).

[25] T. Kamae et al., Phys. Rev. Lett. 38, 468 (1977); T. Kamae et al., Nucl. Phys. B139, 394 (1978); H. Ikeda et al., Phys. Rev. Lett. 42, 1321 (1979); H. Ikeda et al., Nucl. Phys. B172, 509 (1980).

[26] A. S. Bratashevskij et al., Nucl. Phys. B166, 525 (1980); A. S. Bratashevskij et al., Yad. Fiz. 31, 860 (1980) [Sov. J. Nucl. Phys. 31, 444 (1980)]; A. S. Bratashevskij et al., Pis'ma Zh. Eksp. Teor. Fiz. 35, 489 (1982) [JETP Lett. 35, 605 (1982)]; A. S. Bratashevskij et al., Yad. Fiz. 43, 785 (1986) [Sov. J. Nucl. Phys. 43, 499 (1986)]; A. A. Zybalov et al., Nucl. Phys. A533, 642 (1991); V. B. Ganenko et al., Z. Phys. A 341, 205 (1992).

[27] V.P. Barannik et al., Nucl. Phys. A 451, 751 (1986).

[28] A. S. Bratashevskij et al., Pis'ma Zh. Eksp. Teor. Fiz. 34, 410 (1981) [JETP Lett. 34, 389 (1981)]; A. S. Bratashevskij et al., Pis'ma Zh. Eksp. Teor. Fiz. 36, 174 (1982) [JETP Lett. 36, 216 (1982)]; A. S. Bratashevskij et al., Yad. Fiz. 44, 960 (1986) [Sov. J. Nucl. Phys. 44, 619 (1986)].

[29] Y. Kang, P. Erbs, W. Pfeil, and H. Rollnik, Abstracts of the Particle and Nuclear Intersections Conference (MIT, Cambridge, MA, 1990); Y. Kang, Ph.D. thesis, Bonn, 1993.

[30] J. M. Laget, Nucl. Phys. A312, 265 (1978).

[31] W. Liedemann and H. Arenhövel, Nucl. Phys. A465, 573 (1987).

[32] H. Tanabe and K. Ohta, Phys. Rev. C 40, 1905 (1989).

[33] M. Schwamb, H. Arenhövel, and P. Wilhelm, Few-Body Syst. 19, 121 (1995).

[34] S. I. Nagornyi, Yu. A. Kasatkin, and I. K. Kirichenko, Yad. Fiz. 55, 345 (1992) [Sov. J. Nucl. Phys. 55, 189 (1992)]; D. Sivers (private communication).

[35] Note also recent $\Sigma$ asymmetry results from Yerevan: F. Adamian et al., Eur. Phys. J. A 8, 423 (2000).

[36] M. M. Sargsian (private communication).

[37] E. De Sanctis et al., Few-Body Syst. Suppl. 6, 229 (1992); L. A. Kondratyuk et al., Phys. Rev. C 48, 2491 (1993).

[38] E. De Sanctis and L. A. Kondratyuk (private communication).

[39] A. Radyushkin (private communication).

[40] S. J. Brodsky and J. R. Hiller, Phys. Rev. C 28, 475 (1983). 